\title{
Motivating students and reinforcing foundational concepts through effective course design and curriculum integration
}

\section{Barry Shoop}

Barry L. Shoop, "Motivating students and reinforcing foundational concepts through effective course design and curriculum integration," Proc. SPIE 9664, Ninth International Topical Meeting on Education and Training in Optics and Photonics, 966402 (24 October 2005); doi: 10.1117/12.2207785

Event: Ninth International Topical Meeting on Education and Training in Optics and Photonics, 2005, Marseille, France 


\title{
Ref ETOP099
}

\section{Motivating Students and Reinforcing Foundational Concepts Through Effective Course Design and Curriculum Integration}

\author{
Barry L. Shoop
}

\begin{abstract}
s
Effective course design that is focused on system-level applications can effectively enhance student motivation and inquiry-based learning. Deliberate curriculum integration of topical linkages and recurring thematic examples demonstrate course-to-course disciplinary linkages and reinforce foundational concepts. The goal of effective course design is to motivate student interest and learning throughout the course and develop inquiry-based techniques. Curriculum integration includes longitudinal integration of recurring thematic examples that provide opportunities for students to revisit familiar examples with new tools and look at these examples from a different perspective. Integrating these techniques motivates student interest and reinforces foundational concepts through repetition.
\end{abstract}

\section{Summary}

Many undergraduate courses are structured to present underlying theoretical concepts first in the course curriculum and then subsequently, after covering the necessary theoretical concepts, introduce system-level applications. A traditional photonics engineering course, for example, first reviews electromagnetic field theory, addressing essential concepts from geometrical and wave optics followed by an investigation of the interaction of photons with materials. Building upon these fundamental principles, the students then study the operating principles and design considerations of photoemitters, photodetectors, optical waveguides, and optical modulators. Individual devices are then combined in the design, construction and testing of a system - an example being a fiber optic communication link. This approach is often frustrating for the students because it is the applications that motivated them to study the subject and in many cases they have lost focus and interest well-before the applications are covered.

Additionally, concept reinforcement within individual courses and among courses in the curriculum is frequently not deliberate. Students often fail to understand the importance of linkages within and among courses and subjects, and instead view their education as a series of disjoint and unrelated topics and courses. Making conceptual linkages and transferring knowledge from one context to another is a particularly important skill to teach students and, as importantly, is an effective teaching technique. Learning new information is more effective and efficient if the new information is framed within a known context and in fact, deduced from an established knowledge base. Many of the most accomplished and successful scientists and engineers understand new concepts by relating them to foundational theories and framing the new concepts within the context of their own knowledge and experience. In a course like photonics engineering, earlier courses in chemistry, physics, electromagnetic fields and waves, signals and systems, and solid state electronics must all be leveraged to make the most effective use of time, extend previously developed foundational concepts to new applications, and deliberately reinforce those concepts.

Both of these challenges can be overcome by deliberate course and curriculum design. The first can be overcome by designing courses around a system-based application and the second by deliberate integration of the curriculum. The system-based application can be introduced early in the course and used effectively to motivate student-centric, inquiry-based 
education. Integration of the curriculum begins by identifying common foundational themes within and between courses, and highlighting these to students as the topical coverage warrants. Deliberate integration of the curriculum is accomplished by not only identifying the foundational themes through conceptual abstraction, but also, by design of common exemplars.

An example of this type of course design was applied to an introductory photonics engineering course, 19,20 where an emphasis is placed on an inquiry-based investigation of an optical communication system which is introduced in the first lesson of the course and subsequently used as the educational vehicle throughout the remainder of the course. The underlying theory necessary to understand foundational concepts, device behavior and subsystem operation is presented in a just-in-time fashion. The goal of this methodology is to motivate student interest and learning throughout the course and develop inquiry-based techniques. Photonics Engineering is an upper-division course that was taught by studying a fully-functional wavelength division multiplexing (WDM) optical communication system described in Figure 1. Study of a particular subsystem began by allowing students to ask questions about the associated devices of the operational system. Subsequent lessons were then dedicated to answering the student questions and developing the theory necessary to quantify or qualify the answers. A special emphasis was placed on reinforcing concepts with device-specific laboratory exercises. Whenever possible, the linkages were established with material from previous courses. Although this approach sometimes presented difficult concepts early in the semester, requiring a level of abstraction with unanswered questions, the same material was covered in the restructured course as in the previous traditional course.

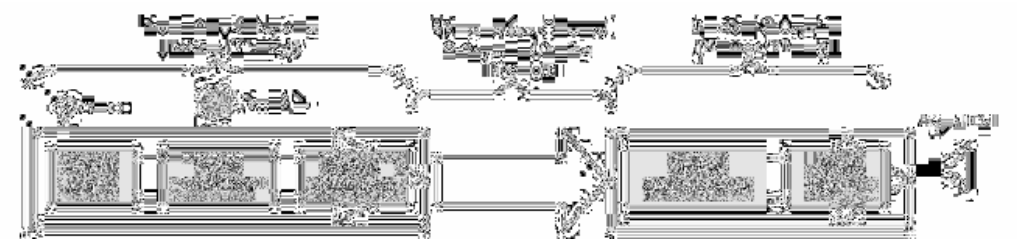

Figure 1. Course flow for wavelength division multiplexed (WDM) optical communication system-based study.

Based on an analysis of assessment tools over a three-year period, the academic performance of the student remained unchanged; the evaluation of knowledge and skill outcomes compared to course objectives reflected a similar level of comprehension for both the traditional and system-based instruction. Despite a reduction in course content, and sometimes incomplete theoretical development, the students were able to perform at an appropriate academic level, especially when asked to integrate photonic devices in operational systems.

Perhaps even more important than academic performance was the increase in the student excitement for the material and the intellectual curiosity of the students. At the beginning of the semester, the average student was reluctant to ask questions or comment on the

\footnotetext{
${ }^{19}$ G. A. Nowak, G. M. Burrow and B. L. Shoop, "System-based Introductory Photonics Engineering Course," Invited Presentation, in Proceedings of Frontiers in Optics, Forum on Education, Rochester, NY (2004).

${ }^{20}$ G. M. Burrow, G. A. Nowak, and B. L. Shoop, "System-based Introductory Photonics Engineering Course - II," in Proceedings of Frontiers in Optic, Forum on Education, Tucson, AZ (2005).
} 
observed characteristics of the operational WDM system. By the end of the term, the intellectual maturity of the student increased to the point that several lessons became freeflowing discussions of the topic.

Additionally, in the Electrical Engineering curriculum at West Point, curriculum integration is accomplished through deliberate curriculum design where topical linkages and recurring thematic examples are used to demonstrate course-to-course disciplinary linkages and reinforce foundational concepts. One example of a deliberate curriculum integration21 theme is one which strives to unify the development of topics such as resonance, filtering, stability, transmission line behavior, and spectral characteristics of lasers in courses such as signals and systems, basic electric circuits, controls, electromagnetic fields, and photonics from mathematical models and analysis techniques associated with second-order linear system response describing damped harmonic oscillators.

\footnotetext{
${ }^{21}$ B. L. Shoop, G. A. Nowak, and L. A. Shay, "Deliberate Longitudinal Curricular Integration: Topical Linkages and Concept Reinforcement," in Proceedings of the 2005 American Society for Engineering Education Annual Conference \& Exposition, Portland, Oregon (2005)
} 